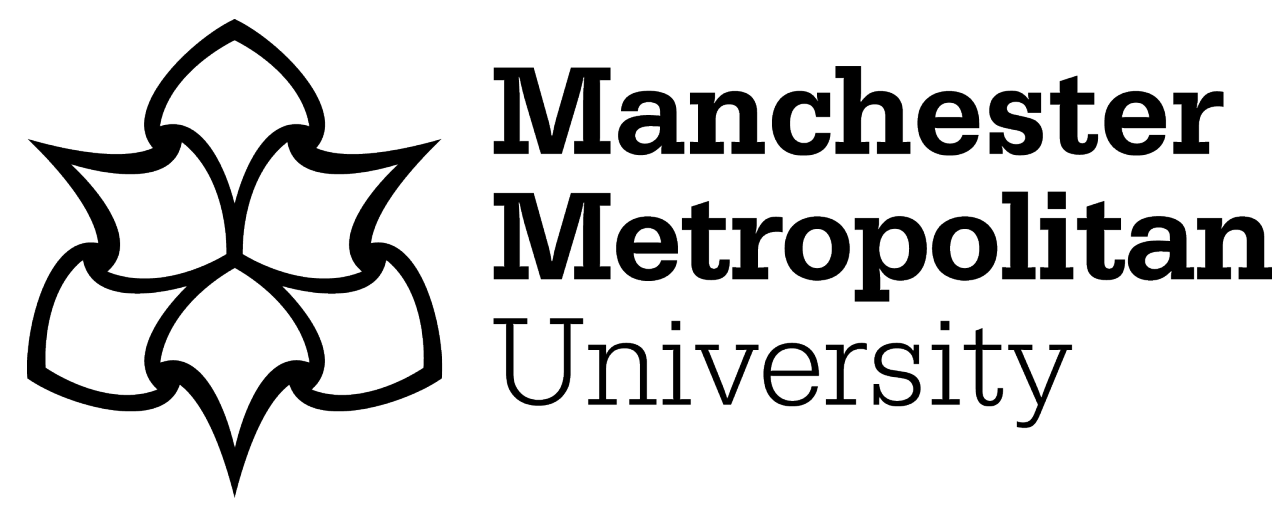

Erfani, R and Kontis, K (2019) MEE-DBD Plasma Actuator Effect on Aerodynamics of a NACA0015 Aerofoil: Separation and 3D Wake. In: Advances in Effective Flow Separation Control for Aircraft Drag Reduction. Computational Methods in Applied Sciences, vol. 2. Springer, pp. 75-92. ISBN 978-3-03029688-9

Downloaded from: https://e-space.mmu.ac.uk/625020/

Version: Accepted Version

Publisher: Springer

DOI: https://doi.org/10.1007/978-3-030-29688-9_4

Please cite the published version 


\title{
MEE-DBD plasma actuator effect on Aerodynamics of a NACA0015 Aerofoil: Separation and 3D Wake
}

\author{
R. Erfani* \\ Manchester Metropolitan University, School of Engineering, Manchester, M1 5GD, UK \\ K. Kontis \\ University of Glasgow, School of Engineering, Glasgow, G12 8QQ, UK
}

\begin{abstract}
Dielectric barrier discharge (DBD) plasma actuators have received considerable attention by many researchers for various flow control applications. Having no moving parts, being light-weight, easily manufacturable, and their ability to respond almost instantly are amongst the advantages which has made them a popular flow control device especially for application on aircraft wings. The new configuration of DBDs which uses multiple encapsulated electrodes (MEE) has been shown to produce a superior and more desirable performance over the standard actuator design. The objective of the current study is to examine the effect of this new actuator configuration on the aerodynamic performance of an aerofoil under leading edge separation and wake interaction conditions. The plasma actuator is placed at the leading edge of a symmetric NACA 0015 aerofoil which corresponds to the location of the leading edge slat. The aerofoil is operated in a chord Reynolds number of $0.2 \times 10^{6}$. Surface pressure measurements along with the mean velocity profile of the wake using pitot measurements are used to determine the lift and drag coefficients, respectively. Particle image velocimetry (PIV) is also utilised to visualise and quantify the induced flow field. The results show improvement in aerodynamic performances of aerofoil under leading edge separation and also facing the wake region.
\end{abstract}

Keywords: Plasma actuator, Dielectric Barrier Discharge (DBD), Flow control, Leading edge separation, Wake interaction

*r.erfani@mmu.ac.uk, DDI: +44 (0) 1612476219 


\section{INTRODUCTION}

Based on the current trend of aircraft development, environmental factors such as noise and emission limits will play a more vital role in future transport aircraft design, driving the need for greener more efficient aircraft. While the aerospace industry is striving to have more and more optimised designs, it is still some way away from the targets set out in the ACARE 2020 vision for $80 \%$ cut in $\mathrm{NO}_{x}$ emissions and $50 \%$ reduction in $\mathrm{CO}_{2}$ emissions. Of the primary objectives of the Clean Sky initiative is achieving a total reduction in aircraft drag of $10 \%$ by reducing the wing drag by $25 \%$. Another goal is the reduction of fuel burnt by $20 \%$. Both the reduction in drag and fuel savings are intertwined since through innovative flow control mechanisms the need for heavy and bulky devices along the wing would be eliminated, leading to lighter aircraft and the lower consumption of fuel and hence, lower emissions.

In comparison, although passive flow control methods are relatively simpler to design and manufacture, they are only effective over a small range. Therefore, when dealing with unsteady motion, such as wake interaction with aerofoils, active flow control is the dominant choice. One of the disadvantageous of active flow control is the requirement of additional power.

The first recorded use of plasma for active flow control was by Velkoff and Ketcham ${ }^{1}$ who used a corona discharge to manipulate the transition point on a flat plate. The use of the 'ionic wind' generated by a corona discharge was the main focus of research attention until the late 1990's. In 1998 a new configuration of electrodes was presented by Roth et al. ${ }^{2}$ that produced a One Atmosphere Uniform Glow Discharge Plasma (OAUGDP). This configuration was able to produce a jet in still air and manipulate the boundary layer of a flat plate.

Plasma actuators require no moving parts in converting electrical energy into kinetic energy, use a simple system structure, can operate over a broad frequency range and can be used instantaneously, making them ideal for flow control purposes. The simple actuator design and ease of construction allows the actuator to be retro-fitted to existing aircraft and are also easy to manufacture and service. Previous studies have shown that dielectric barrier discharge (DBD) plasma actuators are efficient in different flow control applications: boundary layer manipulation, ${ }^{3}$ lift augmentation on a wing section, ${ }^{4}$ transition point manipulation, ${ }^{5}$ 
and in particular in the field of separation control. ${ }^{6-10}$

Their benefits have not been fully utilised on a large scale since the applicability of plasma actuators is limited by the maximum induced velocity it can achieve. Dielectric temperature has significant effect on the strength of the induced velocity. ${ }^{11}$ It was initially shown that breaking down the encapsulated electrode and burying each of the electrodes in a different depth respect to the surface can affect the maximum induced velocity and jet characteristics of the plasma actuator. ${ }^{12,13}$ This type of actuator is known as the multiple encapsulated electrode (MEE) configuration. MEE-DBD plasma actuator has shown its improved performance over the conventional DBD plasma actuator by increasing the induced jet velocity at a lower power consumption. ${ }^{14}$

Two topics which have attracted a great deal of attention from aerodynamicists are: i) aerofoil leading edge flow separation leading to stall, and ii) aerofoil-wake interaction. These two test cases will be used to demonstrate the performance of a MEE-DBD plasma actuator in manipulating the aerodynamic coefficients of a NACA 0015 aerofoil with a chord Reynolds number of $0.2 \times 10^{6}$. The description of the optimum design of MEE-DBD which has been used in the current study has been presented by Erfani et al. ${ }^{15,16}$

\section{A. Leading edge stall}

The stall condition is an undesirable phenomenon where controlling it will improve the aircraft performance by enabling it to take off and land at higher incidences and lower speeds. Controlling the stall angle can be achieved through delaying the leading edge separation on the aerofoil which is usually associated with achieving higher lift magnitudes and lower drag. There are several mechanisms in the field of passive and active flow control to prevent leading edge separation, such as: roughness and trips, vortex generators, 2D and 3D bumps, and dimples are amongst the passive methods (but not limited to) while boundary layer suction, leading edge flap and tangential steady/pulsating blowing piezoelectric actuators, synthetic jets, Lorentz force separation control, oscillating surfaces and membranes are amongst the active ones. All of these methods are designed to enhance the momentum of the flow or remove the low momentum flow close to the surface in order to overcome adverse pressure gradients.

Post and Corke ${ }^{7}$ illustrated the advantage of plasma actuators compared to vortex gener- 
ators. The aerofoil used in their experiment was a NACA $66_{3}-018$ with a maximum Reynolds number of $0.15 \times 10^{6}$. The DBD plasma actuator found to delay the separation by $6^{\circ}$ past the stall angle. He et al. ${ }^{6}$ showed that the leading edge separation control by the use of conventional DBD plasma actuator resulted in an increase in both the maximum lift coefficient and the stall angle of attack by $4^{\circ}$, for NACA 0015 aerofoil with chord Reynolds number of $0.2 \times 10^{6}$. Rethmel et al. ${ }^{10}$ carried out a study with an identical aerofoil and chord Reynolds number flow and observed that the nanosecond pulse driven DBD can extend the stall angle.

\section{B. Aerofoil-Wake interaction}

In aeronautics, most open and closed turbo-machineries, and other engineering disciplines the flow over an aerofoil is influenced by a wake which for instance is originated from the preceding aerofoil. Such a wake can reduce the lift and increase loads on the downstream aerofoil which can create dangerous conditions for flying vehicles. This makes the study of the wake influence on aerodynamic characteristic of the aerofoil necessary. The disturbances in external flow initiate and force transition of boundary layers from laminar to turbulence. Extensive analytical, numerical and experimental studies have been conducted on boundary layer-wake interaction. ${ }^{17-19}$

The aerodynamic characteristics under the influence of the preceding wake have also been studied in relation to the laminar-turbulent transition of the boundary layer over an aerofoil. It is expected that the boundary layer over the aerofoil becomes turbulent through a transition process caused by the interaction between the wake and the boundary layer. ${ }^{20-24}$ In addition, there are some investigations regarding the studies of aerodynamic forces of flying vehicle facing the wake of an aircraft. ${ }^{25,26}$

Kornilov et al. ${ }^{27}$ discussed the interaction between an incompressible two-dimensional turbulent wake produced by a symmetrical aerofoil at incidence and a boundary layer formed on a similar aerofoil immediately downstream. A significant reduction in the level of turbulence was found in the boundary layer of the downstream aerofoil when it is located in the wake periphery. They identified that the outer region of this interaction can be described by simple correlations traditionally used for the wake behind a circular cylinder. Fujisawa et al. ${ }^{20}$ discussed the influence of the circular cylinder wake on the aerodynamic performance of an aerofoil. Their results indicated that due to the interactions between the fluid forces 
and the cylinder wake the drag force decreases and the lift force increases at relatively large angles of attack typically beyond $\alpha=12$ degrees. Their results show an improvement in aerodynamic performance of the aerofoil due to the influence of cylinder wake except for the lift coefficient at $\alpha=9$ to 12 degrees.

\section{PLASMA PHYSICS}

The typical plasma actuator is a linear asymmetric arrangement of two electrodes separated by a dielectric material. One electrode is exposed to the air, while the other is encapsulated in the dielectric. A schematic illustration of such an actuator is depicted in Figure 1 for a standard and MEE-DBD actuator. A high voltage alternating current (a.c.) input, with typical voltages of $2 \mathrm{kV}_{p-p}$ to $60 \mathrm{kV}_{p-p}$ (peak to peak voltage) and frequencies of $300 \mathrm{~Hz}$ to $1 \mathrm{MHz}$ is supplied to the exposed electrode while the encapsulated electrode is earthed. Typically, the plasma actuator is long and thin and is placed spanwise on the aerodynamic surface.

Air ionisation occurs at the edge of the exposed electrode on top of the insulated surface where a region of high electric field potential exists, resulting in the formation of a visible plasma. The plasma is limited to the extent of the covered electrode. ${ }^{28}$ Energetic, relatively high mass ions impart momentum into the neutral air, forming an induced jet. This jet is directed away from the exposed electrode, due to the asymmetric placement of electrodes, and in turn introduces momentum into the flow.

Although the plasma appears as a relatively uniform diffuse discharge to the unaided eye, similar to that shown in Figure 2, optical measurements of the plasma indicate that each half of the voltage cycle consists of the ignition, expansion and quenching of many microdischarges. ${ }^{29,30}$ The charge deposited on the surface reduces the applied electric field at that location. It also shuts down the discharge at macro scales, therefore it is necessary to supply a alternating voltage to maintain a high enough potential gradient to sustain the plasma. The presence of dielectric prevents the formation of an electric arc and spreads the discharges along the dielectric surface almost uniformly.

Figure 3(a) shows the current and alternating voltage trace for a DBD plasma actuator. As the voltage of the exposed electrode becomes more negative electrons are deposited upon the dielectric surface, as shown schematically in Figure 3(b). This stage of the cycle has a 
current discharge that is relatively uniform due to the ease with which electrons can leave the electrode surface between 16 and $41 \mu \mathrm{s}$. During the second part of the cycle, when the voltage becomes increasingly positive, the deposited electrons are attracted back to the exposed electrode as shown in Figure 3(c). During this phase the electrons find it harder to leave the dielectric surface, this behaviour leads to a patchy discharge visible in the current trace between 58 and $83 \mu$ s.

Besides electrons other species such as negative oxygen ions exist also in the plasma, as a result of electron reattachment. ${ }^{31}$ Since ions are larger in mass they can transfer more momentum to the neutral air compared to electrons. It has been shown that the majority of momentum coupling happens when the voltage is becoming more negative and the electrons and negative ions are attracted to the dielectric surface. ${ }^{32,39}$

\section{APPARATUS AND INSTRUMENTATION}

\section{A. Wind tunnel}

The experiments were conducted in one of the subsonic wind tunnels at the Aero-Physics Laboratory in the school of MACE at The University of Manchester. The facility is an open-return low subsonic 'blower' with a $0.455 \mathrm{~m}$ square cross section by $1.4 \mathrm{~m}$ long test section. The ceiling and side walls are made of optical grade perspex to allow optical access for photography and visualisation. Turbulence in the test section is reduced by the presence of honeycombs located upstream of the test section. The turbulent intensity in the test section over the range of velocities used in the experiment is approximately $0.24 \%$. The wind tunnel speed was monitored using a pitot-static tube placed upstream of the models mounted within the test section.

\section{B. Aerofoil}

The aerofoil used in the experiments was a NACA 0015 constructed from perspex with a chord length, c, of $0.24 \mathrm{~m}$ and span of $0.40 \mathrm{~m}$. The characteristics of this aerofoil are well documented in the literature and it exhibits well-behaved leading edge separation at high angles of attack. For minimising the end effects, end plates are mounted on the sides of the aerofoil made from optical grade perspex to allow visual access for flow visualisation 
and measurements. The end plates were $0.685 \times 0.457 \times 0.01 \mathrm{~m}$ (length $\times$ height $\times$ thickness) . The leading and trailing ends of the end plates were machined to have a $5 \mathrm{~mm}$ radius. The maximum blockage of the aerofoil which occurs at the highest incidence was estimated to be $3.9 \%$. Therefore, tunnel blockage effect on the aerodynamic coefficients considered minimal and no correction in the measuremnets taken were necessary.

\section{Cylinder}

At the working chord Reynolds number of $0.2 \times 10^{6}$, the frequency of the wake of the aerofoil at zero incidence is measured as $120 \mathrm{~Hz}$ and a Strouhal number, $\mathrm{St}=\mathrm{fD} / \mathrm{U}_{\infty}$, of the cylinder estimated to be 0.2 , where $\mathrm{f}$ is the frequency of the wake, $\mathrm{D}$ is the diameter of the cylinder and $\mathrm{U}_{\infty}$ is the free-stream velocity. ${ }^{33-35}$ Therefore, a cylinder which can generate a frequency similar to that of the aerofoil should have a diameter of $0.02 \mathrm{~m} .^{20}$

The vortex shedding of the cylinder depends strongly on its diameter and the distance between the cylinder and the aerofoil. This critical distance is found by Luk et al. ${ }^{36}$ to be equal to the length of the vortex formation region of the circular cylinder measured without the presence of aerofoil, and is 1.5 times of the diameter of the cylinder behind it. The cylinder is placed between two end plates at $0.2 \mathrm{~m}$ upstream of the aerofoil.

\section{Plasma actuator}

The configuration of plasma actuator examined in the current investigation is shown in the schematic of Figure $4 .{ }^{15}$ The actuator is placed at the leading edge while the interface of the exposed electrode and the first covered electrode is located at $\mathrm{x} / \mathrm{c}=0$. The encapsulated electrodes are aligned so that there is no offset between the edges of successive electrodes. All the electrodes are tinned copper foils, $74 \mu \mathrm{m}$ thick and $200 \mathrm{~mm}$ in length, in the spanwise direction. Layered Kapton tape was used as a dielectric material with each layer having a $60 \mu \mathrm{m}$ thickness. Dimensions and placement of the electrodes are also provided in Figure 4. The offset listed in figure is the distance measured from the downstream edge of the exposed electrode. To have a uniform plasma along the span, a small amount of overlap is applied between the downstream edge of the exposed electrode and upstream edge of the first encapsulated one. 
The actuator was controlled by a LabView program where the wave shape, driving frequency, modulation frequency and corresponding duty cycles are controlled. This signal was provided via National Instruments PCI-6713 to a control circuit board that was also connected to a Volkraft 3610 power supply capable of outputting $360 \mathrm{~W}$. The powered control signal is then connected to a transformer cascade which provides the high voltage signal to drive the actuator. The transformer is capable of a maximum $40 \mathrm{kV}_{p-p}$ at driving frequencies up to $30 \mathrm{kHz}$.

The voltage supplied to the exposed electrode is measured using a LeCroy PPE-20 kV high voltage probe while the current is monitored using a current probe attached to the transformer cascade output. The two probes are connected to a Picoscope 3206, $250 \mathrm{MHz}$ oscilloscope connected to a PC so the output signals can be monitored and recorded.

The actuator spanned most of the width of the leading edge of aerofoil. A narrow gap was left at the centre line due to the presence of pressure taps along chordwise direction. However, both sides were electrically connected. A recess equal to the thickness of the actuator had been cut from the aerofoil in order to produce a flush surface after mounting the actuator without changing the profile shape of the aerofoil.

\section{E. Pressure measurements}

A total of 23 surface pressure ports with an internal diameter of $1.5 \mathrm{~mm}$ were aligned in the flow direction at the half-span location of the aerofoil. The pressure tubes were passed through the side wall of the test section and connected to a scanning pressure valve that selectively connected each pressure port to a single pressure transducer.

A separate rake of pitot probes was mounted on a traversing mechanism and located downstream of the aerofoil at its spanwise centre line. Discrete points, with $2.54 \mathrm{~mm}$ distance between each probe, were sampled across the wake to determine the total pressure profile. The rake was placed one chord length downstream of the trailing edge in order to be far from any recirculation region that might exist in the separated flow.

For all pressure measurements, data was recorded for 4 seconds at a sampling rate of 10 $\mathrm{kHz}$ and averaged for both lift and drag measurements. 


\section{F. Particle image velocimetry (PIV)}

Particle image velocimetry (PIV) uses the displacement of particles to determine various flow field parameters. A laser beam is manipulated into a thin sheet using an arrangement of lenses to illuminate the region of interest. Using two successive laser pulses separated by a known time, statistical analysis can be performed to measure the instantaneous velocity of the tracer particles captured with a high-speed camera. The PIV measurements were performed using a TSI system with an NDYAG 532 nm, Litron 120 mJ, pulsed laser. The laser was operated at a rate of $1 \mathrm{kHz}$. In these experiments the duration between pulses was set at $20 \mu \mathrm{s}$. This value is set based on the field of view size and expected induced velocity, of the order of $10 \mathrm{~m} / \mathrm{s}$. The laser was delivered using a laser arm mounted above the actuator to produce a laser sheet that ran along the centre-line of the aerofoil span with a bit of offset. The flow was seeded with light-scattering olive oil particles with a size of approximately $1 \mu \mathrm{m}$.

\section{RESULTS AND DISCUSSION}

Figure 5 illustrates the comparison between mean velocity profiles $50 \mathrm{~mm}$ from the exposed electrode working in quiescent conditions of a standard DBD and optimised MEEDBD actuator. The measurements were obtained from PIV experiments on actuators mounted on a flat surface. The actuators were operated at $15 \mathrm{kV}_{p-p}$ with a driving frequency of $10 \mathrm{kHz}$ using a sine wave input and 100\% duty cycle. This driving frequency provides the actuator with a clean sinusoidal input signal not experienced at the other frequencies. The power consumption of the actuator was approximately $15 \mathrm{~W}$. Identical settings are used on the aerofoil. As it is evident from the plot, the MEE-DBD actuator creates a faster induced jet with a greater jet thickness. This behaviour was also observed at different locations along the actuator.

\section{A. Leading edge plasma actuator}

The results in this section document the ability of the leading edge MEE-DBD plasma actuator to reattach the flow at high angles of attack. Studies have shown that placing the actuator at the leading edge of an aerofoil, at the point of separation, has the best effect 
on the control of the leading edge separation. ${ }^{7,37}$ The presented results are taken at chord Reynolds number of $0.2 \times 10^{6}$ which enable us to compare with literature and in particular the results of Post and Corke, ${ }^{7}$ He et al. ${ }^{6}$ and Rethmel et al. ${ }^{10}$ which are taken at a similar Reynolds number. The effectiveness of MEE plasma actuator in separation flow control was evaluated on the basis of lift enhancement and drag reduction.

The pressure coefficient distribution and the momentum deficit in the wake of the aerofoil are used to assess the lift and drag coefficients for different angles of attack, with the plasma actuator off and actuator on.

Figure 6 shows the variation of lift and drag coefficients $\left(\mathrm{C}_{l}\right.$ and $\left.\mathrm{C}_{d}\right)$ at various incidences with the actuator off (baseline) and on. These values are calculated based on the procedure given by Erfani et al. ${ }^{38,39}$ At low angles of attack, the baseline values of $\mathrm{C}_{l}$ are in good agreement with thin aerofoil theory, namely $d \mathrm{C}_{l} / d \alpha=0.12$. The shape of the drag polar and $\mathrm{C}_{l}$ are also consistent with the results mentioned in literatures for this family of aerofoils. ${ }^{7,40}$

With the actuator off, a sudden reduction in lift coefficient after $14^{\circ}$ and an increase in drag at $\mathrm{C}_{l}=1.2$ corresponds to the static stall angle which is accompanied by flow separation from the leading edge. The significant improvement that is produced when the plasma actuator is switched on is the increase in stall angle from $14^{\circ}$ to $22^{\circ}$, that is $8^{\circ}$ past the natural stall angle of attack. By controlling the leading edge separation, the drag is also reduced, as can be seen in Figure 6(b). Comparing lift to drag ratios between the on and off cases shows a maximum improvement of 52 times for incidences between $15^{\circ}$ to $22^{\circ}$. This value is greater than what has been presented in the literature for the standard DBD configuration. 6,7

The pressure distributions for post-stall angles of $17^{\circ}$ and $22^{\circ}$ are shown in Figure $7(\mathrm{a})$, $17^{\circ}$ is chosen since it corresponds to the largest lift to drag ratio and $22^{\circ}$ is chosen to examine the drop in $\mathrm{C}_{l}$ observed at this incidence. When the actuator is off, the flow separates at the leading edge without reattaching, creating a large wake which extends beyond the trailing edge. This behaviour results in a constant magnitude of pressure coefficient, $\mathrm{C}_{p}$, along the chord. When the actuator is switched on, the suction pressure is recovered and the separation is suppressed up to $\mathrm{x} / \mathrm{c}=0.6$ and $\mathrm{x} / \mathrm{c}=0.3$ for angles of $17^{\circ}$ and $22^{\circ}$, respectively. The fact that the separation point is delayed only up to $\mathrm{x} / \mathrm{c}=0.3$ at $22^{\circ}$ incidence, justifies the drop in lift coefficient observed in Figure 6(a). Figure 7(b) shows mean velocity profile within the wake. It is observed that the wake region is narrower when the actuator is on 
which clearly illustrates the ability of plasma actuator in preventing or delaying the onset of separation. It is because of the introduction of high momentum jet to the surface at the point of separation which overcomes the adverse pressure gradient and therefore decreases the width of the separation region by approximately a third.

Figure 8 compares the PIV data obtained for incidences of $17^{\circ}$ and $22^{\circ}$ with actuators off and on. A white PIV mask is applied to the images to remove any bad vectors close to the aerofoil surface. These bad vectors result from the reflection of the laser beam from the surface. Due to the large area under investigation and the lack of illumination size of laser beam, only the region in the immediate vicinity of the separation zone had adequate illumination. For the actuator off case the measurements verify that the drop in lift at higher angles of attack is due to the leading edge flow separation. The flow separation starts from the leading edge and covers the entire suction surface of the aerofoil. For the plasma on case, at incidence of $22^{\circ}$ the separation point corresponds to approximately $\mathrm{x} / \mathrm{c}=0.3$. However, due to the limited illumination size it was not possible to concur that the rear part of the reattachment zone for $17^{\circ}$ occurs at $\mathrm{x} / \mathrm{c}=0.6$.

\section{B. Aerofoil-wake interaction}

The results in this section examine the effects of the leading edge MEE-DBD plasma actuator on the aerodynamic performance of the aerofoil at chord Reynolds number of $0.2 \times 10^{6}$, whilst in the wake of a circular cylinder.

The lift and drag coefficients of the aerofoil at various angles of attack facing the cylinder wake are presented in Figure 9(a) and Figure 9(b), respectively. The lift and drag coefficients of the plane aerofoil are also shown in the figure as the baseline case for comparative purposes. With the actuator in off mode, it is observed that up to the natural stall angle of the aerofoil $\alpha=14^{\circ}$, the presence of the cylinder wake has an adverse effect on the performance of the aerofoil by reducing the lift and increasing the drag. However, at higher incidences the presence of the cylinder wake suppresses the stall and reduced the drag.

Figure 9 also includes the results for plasma on case. In all angles of attack, the actuator on case shows an increase in $\mathrm{C}_{l}$ compared to the actuator off case. Up to $\alpha=14^{\circ}$ the drag coefficient does not change with increasing incidence for all cases, with the actuator on leading to a reduced level of drag compared to the actuator off case. After $\alpha=14^{\circ}$ a 
significant rise in drag coefficient is observed for the baseline case whilst the presence of the cylinder wake suppresses the maximum drag coefficient. The drag coefficient begins to increase after $17^{\circ}$ for the cylinder wake case, but still does not approach the results without the cylinder case. This increase is believed to be due to the flow separation and formation of separation region at the trailing edge.

The pressure distribution on the aerofoil surface for a pre-stall angle of $11^{\circ}$, and a post stall angle of $17^{\circ}$ is shown in Figures 10(a) and (b), respectively. At $11^{\circ}$ incidence the pressure distribution shows a reduction in pressure on the lower surface and increased pressure on the upper surface of the aerofoil compared to the baseline measurements of the aerofoil. No formation of a separated region was detected from the $\mathrm{C}_{p}$ curves in this range of prestall angles of attack. This justifies the constant values of $\mathrm{C}_{d}$ observed in Figure $9(\mathrm{~b})$ for different angles. For the $17^{\circ}$ post stall case, the pressure distribution on the upper surface reveals a large decrease in pressure with the presence of the cylinder wake. This indicates an attached flow pattern on the surface due to the velocity fluctuations of the cylinder wake and its interaction with the boundary layer over the aerofoil. The separation of the boundary layer on the suction side is removed and a turbulent boundary layer forms over the surface. Flow over the lower surface of the aerofoil is also promoted to a turbulent state due to the influence of the cylinder wake which shows itself as a reduction in pressure values in Figure 10.

\section{CONCLUSIONS AND FUTURE WORK}

The influence of MEE-DBD plasma actuator proposed by Erfani et al. ${ }^{15}$ on the aerodynamic performance of a NACA 0015 aerofoil has been investigated in two test cases: leading edge separation control, and aerofoil-wake interaction. Both cases were studied in a chord Reynolds number of $0.2 \times 10^{6}$. Overall, the new plasma actuator configuration produced an improvement in aerodynamic coefficients in both test cases by increasing the lift and reducing the drag.

The stall angle was increased by $8^{\circ}$ past the $\alpha_{\text {stall }}$ angle of $14^{\circ}$ by delaying the leading edge separation. Using the conventional DBD plasma actuator and working at a similar Reynolds number with a NACA 0015 aerofoil, He et al. ${ }^{6}$ was able to increase the stall angle up to $18^{\circ}$ which was $4^{\circ}$ past the $\alpha_{\text {stall }}$ angle. Using a NACA $66_{3}-018$ aerofoil and chord 
Reynolds number of $0.15 \times 10^{6}$, Post and Corke $^{7}$ were able to only achieve an increase of $6^{o}$ past the $\alpha_{\text {stall }}$ angle of $14^{\circ}$.

At high angles of attack, due to the wake excitation of the cylinder wake, the separation region at the suction side of the aerofoil was restrained, leading to the suppression of the stall angle. However, at all incidences the MEE actuator was able to increase the magnitude of the lift coefficient and decrease the level of drag coefficient.

Since a plasma actuator is designed to produce a steady two-dimensional wall jet in the flow direction on the upper surface, the pressure distribution on the lower surface remains unaffected. It would be an interesting study to examine the effect of the plasma actuators placed on the lower surface of the aerofoil. 
${ }^{1} \mathrm{H}$. Velkoff and J. Ketcham. Effect of an electrostatic field on boundary layer transition. AIAA Journal, 6(7):1381-1383, 1968.

2 D.M. Roth, J.R. Sherman and S.P. Wilkinson. Boundary layer flow control with a one atmosphere uniform glow discharge surface plasma. In 36th Aerospace Sciences Meeting 8 Exhibit, Reno, 1998.

3 D.F. Opaits, D.V. Roupassov, S.M. Starikovskaia, A.Y. Starikovskii, I.N. Zavialov, and S.G. Saddoughi. Plasma control of boundary layer using low-temperature non-equilibrium plasma of gas discharge. AIAA Journal, 1180(43):10-13, 2005.

4 T.C. Corke, D.A. Cavalieri, and E. Matlis. Boundary-layer instability on sharp cone at Mach 3.5 with controlled input. AIAA Journal, 40:1015-1018, 2002.

5 S. Grundmann and C. Tropea. Delay of Boundary-Layer Transition Using Plasma Actuators. In 46th AIAA Aerospace Sciences Meeting and Exhibit, Paper Number AIAA-2008-1369, 2008.

${ }^{6}$ C. He, T.C. Corke, and M.P. Patel. Plasma flaps and slats: an application of weakly ionized plasma actuators. Journal of Aircraft, 46(3):864-873, 2009.

7 M.L. Post and T.C. Corke. Separation control on high angle of attack airfoil using plasma actuators. AIAA Journal, 42(11):2177-2184, 2004.

8 J. Huang, T.C. Corke, and F.O. Thomas. Plasma actuators for separation control of low-pressure turbine blades. AIAA Journal, 44(1):51-57, 2006.

9 R Ruisi, H Zare-Behtash, K Kontis, and R Erfani. Active flow control over a backward-facing step using plasma actuation. Acta Astronautica, 126:354-363, 2016.

10 C. Rethmel, J. Little, K. Takashima, A. Sinha, I. Adamovich, and M. Samimy. Flow separation control over an airfoil with nanosecond pulse driven dbd plasma actuators. In 49th AIAA Aerospace Sciences Meeting including the New Horizons Forum and Aerospace Exposition, Orlando, Florida, Paper Number AIAA-2011-487, 2011.

11 R. Erfani, H. Zare-Behtash, and K. Kontis. Plasma actuator: influence of dielectric surface temperature. Experimental Thermal and Fluid Science, 42:258-264, 2012.

12 C. Hale, R. Erfani, and K. Kontis. Plasma actuators with multiple encapsulated electrodes to influence the induced velocity. In 48th AIAA Aerospace Sciences Meeting Including the New Horizons Forum and Aerospace Exposition, AIAA-2010-1223, 2010. 
13 C. Hale, R. Erfani, and K. Kontis. Plasma actuators with multiple encapsulated electrodes to influence the induced velocity : Further configurations. In 40th Fluid Dynamics Conference and Exhibit, AIAA-2010-5106, number 2010-5106, 2010.

14 R. Erfani, C. Hale, and K. Kontis. The Influence of Electrode Configuration and Dielectric Temperature on Plasma Actuator Performance. In 49th AIAA Aerospace Sciences Meeting including the New Horizons Forum and Aerospace Exposition, Orlando, AIAA-2011-955, 2011.

15 R. Erfani, T. Erfani, S.V. Utyuzhnikov, and K. Kontis. Optimisation of multiple encapsulated electrode plasma actuator. Aerospace Science and Technology, 26(1):120-127, 2013.

16 R. Erfani, T. Erfani, C. Hale, K. Kontis, and S.V. Utyuzhnikov. Optimization of Induced Velocity for Plasma Actuator with Multiple Encapsulated Electrodes using Response Surface Methodology. In 49th AIAA Aerospace Sciences Meeting including the New Horizons Forum and Aerospace Exposition, Orlando, AIAA-2011-1206, 2011.

17 L.C. Squire. Interactions between wakes and boundary-layers. Progress in Aerospace Sciences, 26(3):261-288, 1989.

18 T.L. Doligalski and J.D.A. Walker. The boundary layer induced by a convected two-dimensional vortex. Journal of Fluid Mechanics, 139(-1):1-28, 1984.

19 I.S. Gartshore, P.A. Durbin, and J.C.R. Hunt. The production of turbulent stress in a shear flow by irrotational fluctuations. Journal of Fluid Mechanics, 137(-1):307-329, 1983.

20 Y. Takagi, N. Fujisawa, T. Nakano, and A. Nashimoto. Cylinder wake influence on the tonal noise and aerodynamic characteristics of a NACA0018 airfoil. Journal of Sound and Vibration, 297(3-5):563-577, 2006.

21 H. Pfeil, R. Herbst, and T. Schroder. Investigation of the laminar-turbulent transition of boundary layers disturbed by wakes. In American Society of Mechanical Engineers, International Gas Turbine Conference and Exhibit, 27 th, London, England, 1982.

${ }^{22} \mathrm{X}$. Liu and W. Rodi. Experiments on transitional boundary layers with wake-induced unsteadiness. Journal of Fluid Mechanics Digital Archive, 231:229-256, 2006.

23 N.K. Kyriakides, E.G. Kastrinakis, S.G. Nychas, and A. Goulas. Aspects of flow structure during a cylinder wake-induced laminar/turbulent transition. AIAA Journal, 37(10):1197-1205, 1999.

24 R. Mailach and K. Vogeler. Wake-induced boundary layer transition in a low-speed axial compressor. Flow, Turbulence and Combustion, 69(3):271-294, 2002.

25 A.W. Bloy, M.G. West, and K. Lea. Lateral aerodynamics interference between tanker and 
receiver in air-to-air refueling. Journal of Aircraft, 30(5):705-710, 1993.

26 J.D. Iversen and S. Bernstein. Trailing vortex effects on following aircraft. Journal of Aircraft, 11:60, 1974.

27 V.I. Kornilov, G. PAILHAS, and B. AUPOIX. Airfoil-boundary layer subjected to a twodimensional asymmetrical turbulent wake. AIAA Journal, 40(8):1549-1558, 2002.

28 C.L. Enloe, T.E. McLaughlin, R.D. VanDyken, K.D. Kachner, E.J. Jumper, T.C. Corke, M. Post, and O. Haddad. Mechanisms and responses of a single dielectric barrier plasma actuator: geometric effects. AIAA Journal, 42(3):595-604, 2004.

29 R. Erfani, H. Zare-Behtash, and K. Kontis. Influence of shock wave propagation on dielectric barrier discharge plasma actuator performance. Journal of Physics D: Applied Physics, $45: 225201,2012$.

30 C.L. Enloe, T.E. McLaughlin, R.D. VanDyken, K.D. Kachner, E.J. Jumper, and T.C. Corke. Mechanisms and responses of a single dielectric barrier plasma actuator: plasma morphology. AIAA Journal, 42(3):589-594, 2004.

31 Z. Fang, J. Lin, X. Xie, Y. Qiu, and E. Kuffel. Experimental study on the transition of the discharge modes in air dielectric barrier discharge. Journal of Physics D: Applied Physics, 42:085203, 2009.

32 C.L. Enloe, M.G. McHarg, and T.E. McLaughlin. Time-correlated force production measurements of the dielectric barrier discharge plasma aerodynamic actuator. Journal of Applied Physics, 103:073302, 2008.

33 M.M. Zdravkovich. Flow Around Circular Cylinders: Fundamentals. Oxford science publications. Oxford University Press, 1997.

34 T. Nakagawa. A formation mechanism of alternating vortices behind a circular cylinder at high reynolds number. Journal of Wind Engineering and Industrial Aerodynamics, 25(1):113-129, 1986.

35 A. Roshko. Experiments on the flow past a circular cylinder at very high reynolds number. Journal of Fluid Mechanics, 10(03):345-356, 1961.

36 K.F. Luk, R.M.C. So, S.C. Kot, Y.L. Lau, and R.C.K. Leung. Airfoil Vibration Due to Upstream Alternating Vortices Generated by a Circular Cylinder. ASME Applied Mechanics DivisionPublication-AMD, 253(A):79-88, 2002.

37 J.R. Roth and X. Dai. Optimization of the aerodynamic plasma actuator as an electrohydrody- 
namic (EHD) electrical device. In 44th AIAA Aerospace Sciences Meeting and Exhibit, Reno, Paper Number AIAA 2006-1203, pages 9-12, 2006.

38 R. Erfani, C. Hale, and K. Kontis. Flow Control of a NACA 0015 Airfoil in a Turbulent Wake Using Plasma Actuators. In 50th AIAA Aerospace Sciences Meeting including the New Horizons Forum and Aerospace Exposition, AIAA-2012-187, 2012.

39 R. Erfani, H. Zare-Behtash, C. Hale, and K. Kontis. Development of dbd plasma actuators: the double encapsulated electrode. Acta Astronautica, 109:132-143, 2015.

40 I.H. Abbott and A.E.V. Doenhoff. Theory of wing sections, including a summary of airfoil data. Dover books on physics and chemistry. Dover Publications, 1959. 


\section{List of Figures}

1 Typical configurations of standard DBD (a), and MEE-DBD plasma actuators

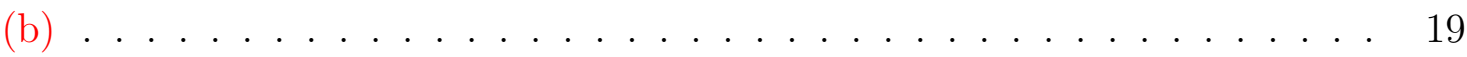

2 Photograph of plasma created on a DBD plasma actuator . . . . . . . . . . . 19

3 Typical Voltage and current trace of a DBD plasma actuator (a), when electrons leave the exposed electrode (b), some deposited electrons returning (c)(subfigures (b) and (c) are not to scale) . . . . . . . . . . . 20

4 Aerofoil actuator configuration at the leading edge (figure not to scale) . . . 20

5 Mean velocity profiles obtained with $15 \mathrm{kV}_{p-p}$ and $10 \mathrm{kHz}$ at $\mathrm{x}=50 \mathrm{~mm}$. 20

6 Lift coefficient (a), and drag polar (b) of the aerofoil for plasma on and off

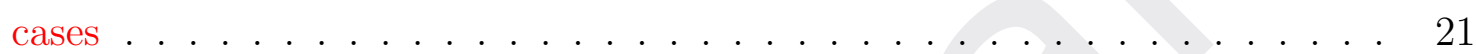

7 Pressure distribution on the aerofoil surface (a), mean velocity profile of the aerofoil wake at $\alpha=17^{\circ}(\mathrm{b}) \ldots \ldots \ldots \ldots . \ldots \ldots 22$

8 Velocity contours on the leading edge portion of the upper surface of the aerofoil at $\alpha=$, (a) $17^{\circ}$, (b) $22^{0} \ldots \ldots \ldots \ldots \ldots$

9 Lift coefficient (a), and drag coefficient (b) . . . . . . . . . . . . 24

10 Pressure distribution on aerofoil surface for $\alpha=$ (a) $11^{\circ}$ (pre-stall), and (b) $17^{\circ}$ (post stall $\ldots \ldots \ldots \ldots \ldots \ldots$. . . . . . . . . . . . . . . . . . . . . . . 

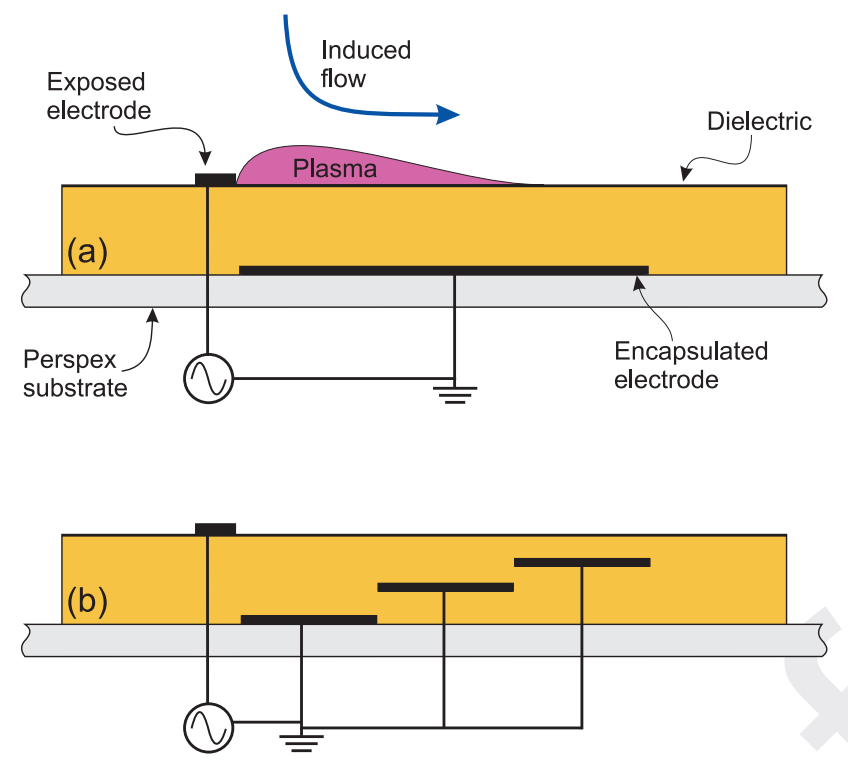

FIG. 1: Typical configurations of standard DBD (a), and MEE-DBD plasma actuators (b)

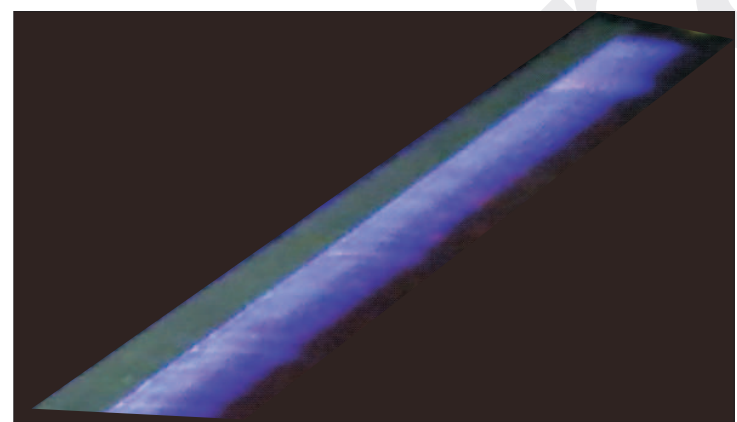

FIG. 2: Photograph of plasma created on a DBD plasma actuator 

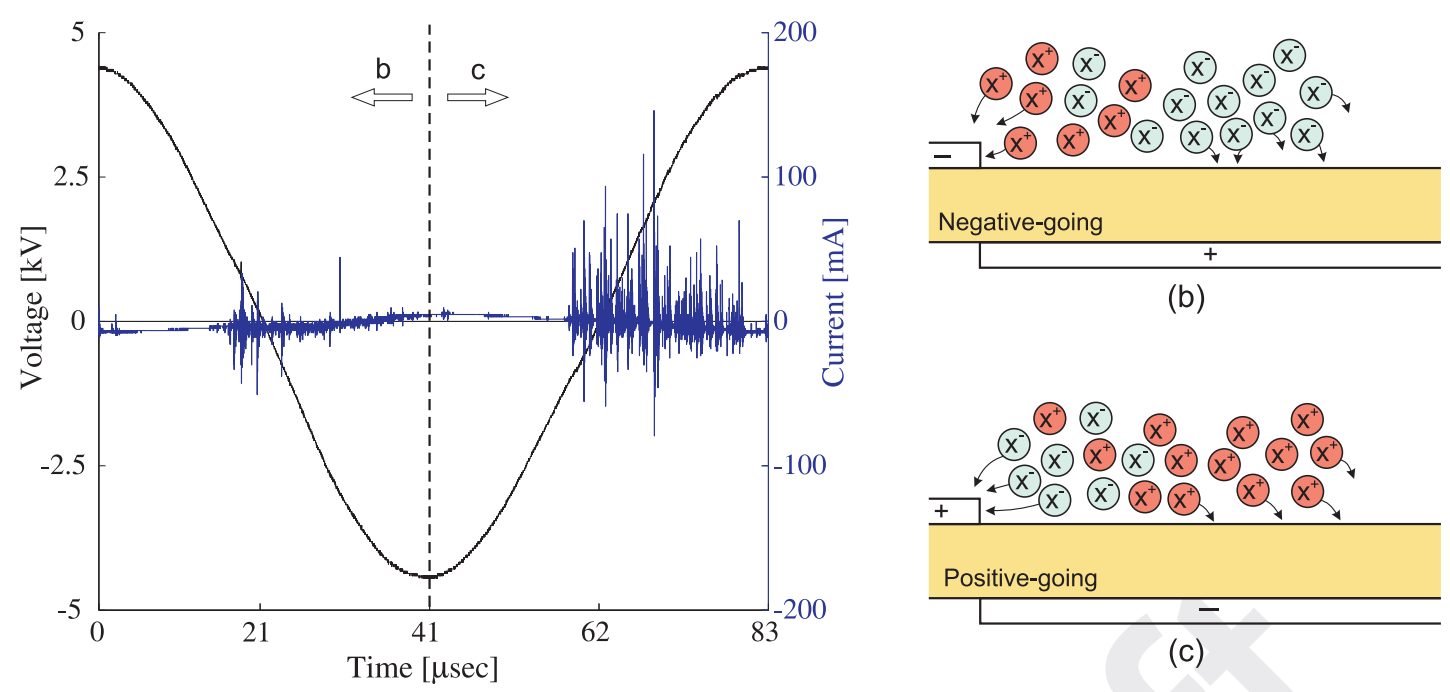

(b)

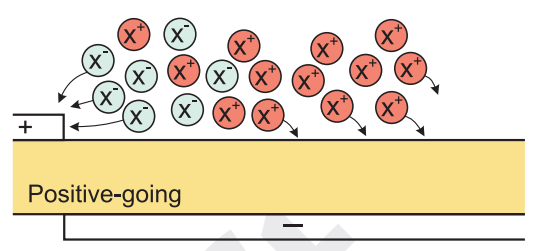

(c)

(a)

FIG. 3: Typical Voltage and current trace of a DBD plasma actuator (a), when electrons leave the exposed electrode (b), some deposited electrons returning (c)(subfigures (b) and (c) are not to scale)

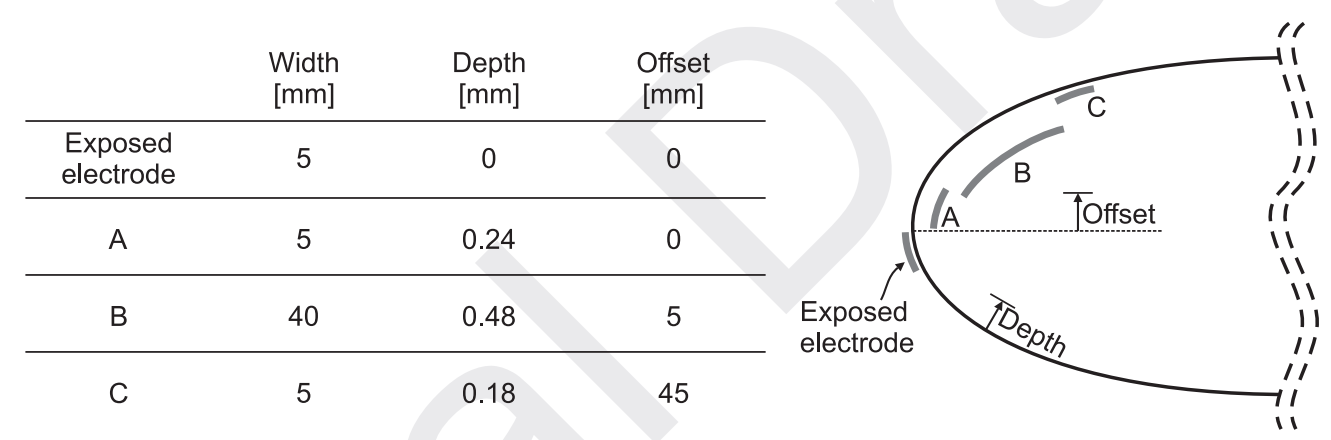

FIG. 4: Aerofoil actuator configuration at the leading edge (figure not to scale)

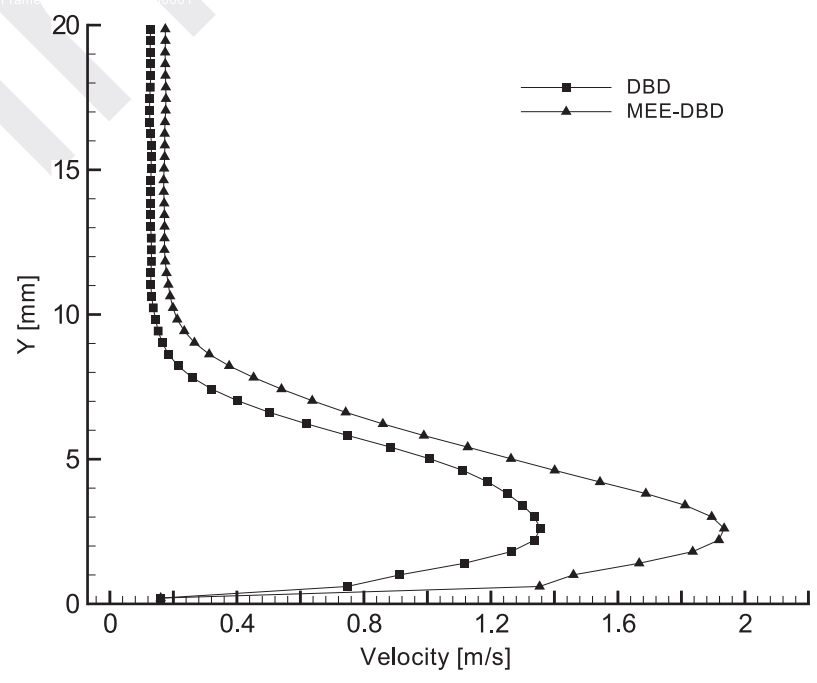

FIG. 5: Mean velocity profiles obtained with $15 \mathrm{kV}_{p-p}$ and $10 k H z$ at $\mathrm{x}=50 \mathrm{~mm}$ 


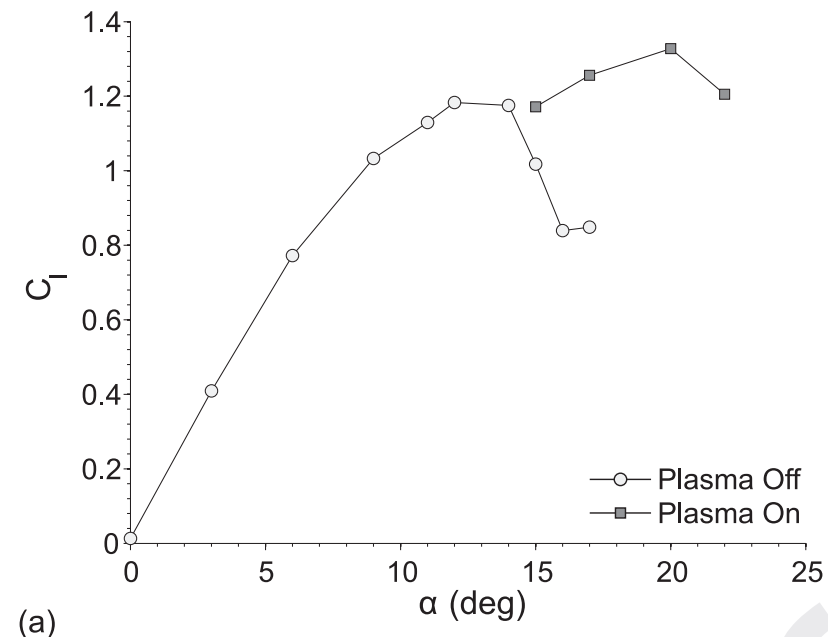

(a)

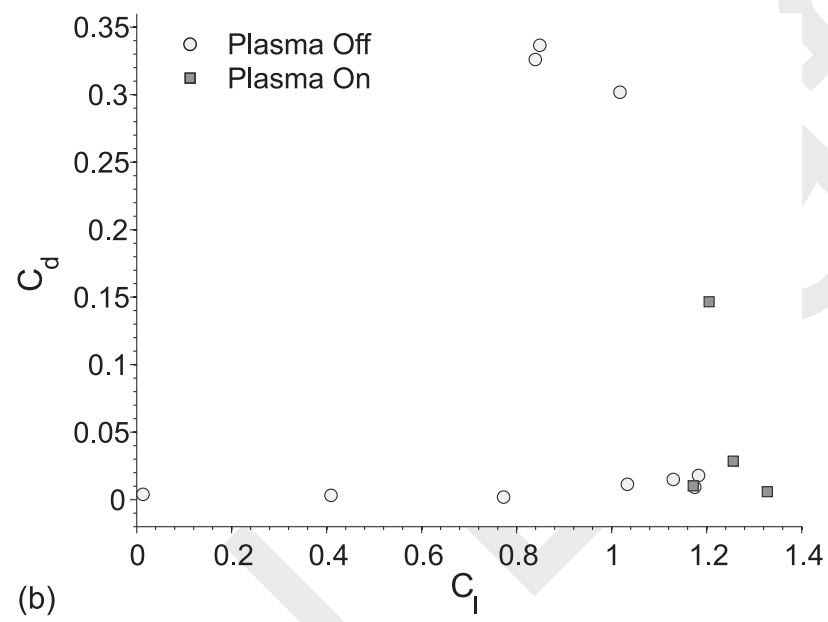

FIG. 6: Lift coefficient (a), and drag polar (b) of the aerofoil for plasma on and off cases 

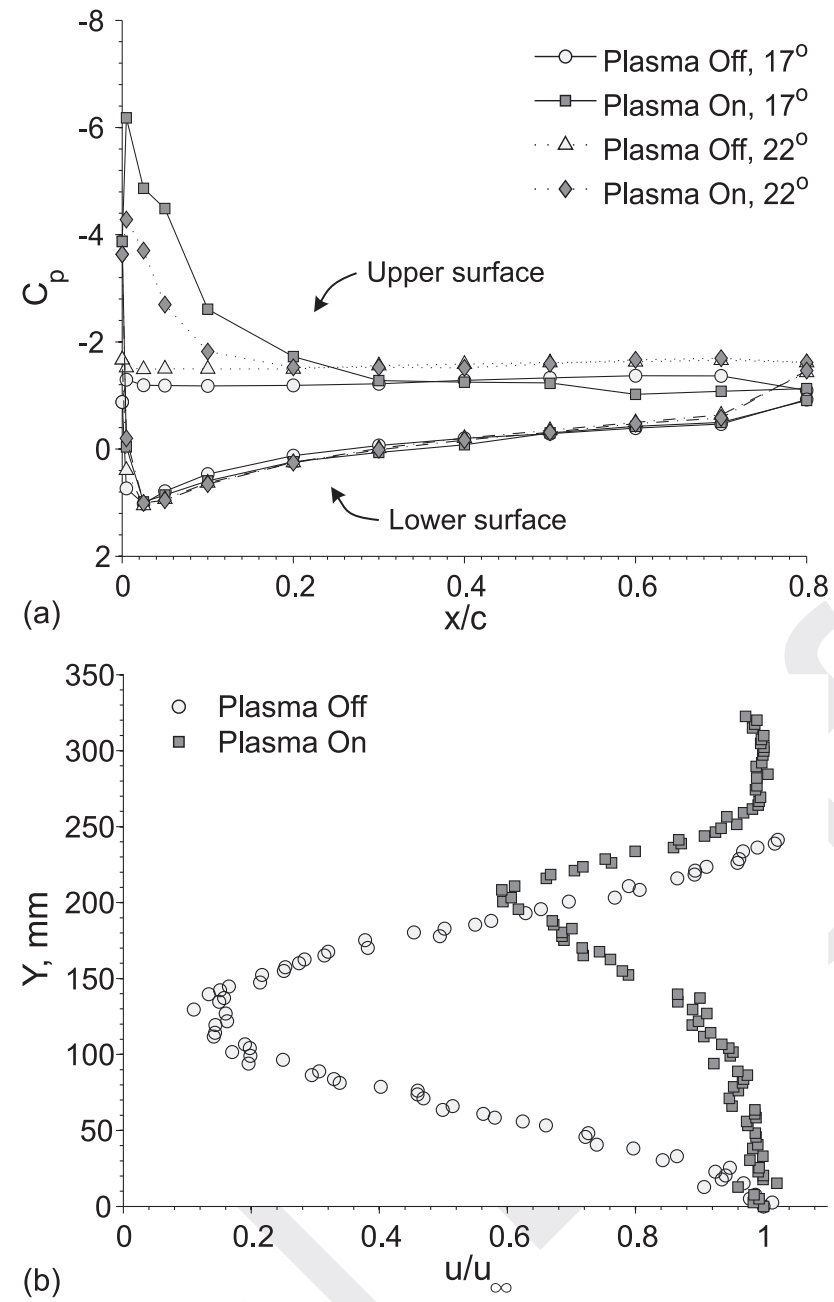

FIG. 7: Pressure distribution on the aerofoil surface (a), mean velocity profile of the aerofoil wake at $\alpha=17^{\circ}$ (b) 

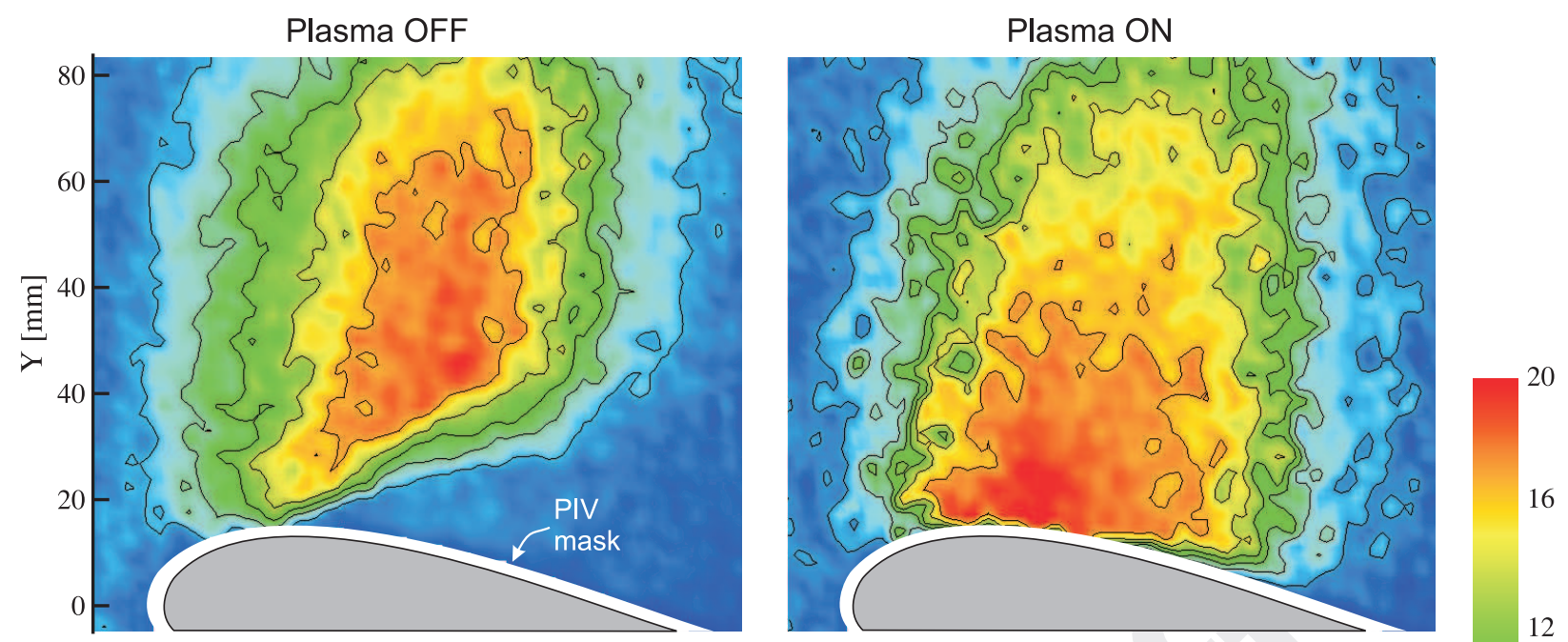

(a) $\alpha=17^{\circ}$
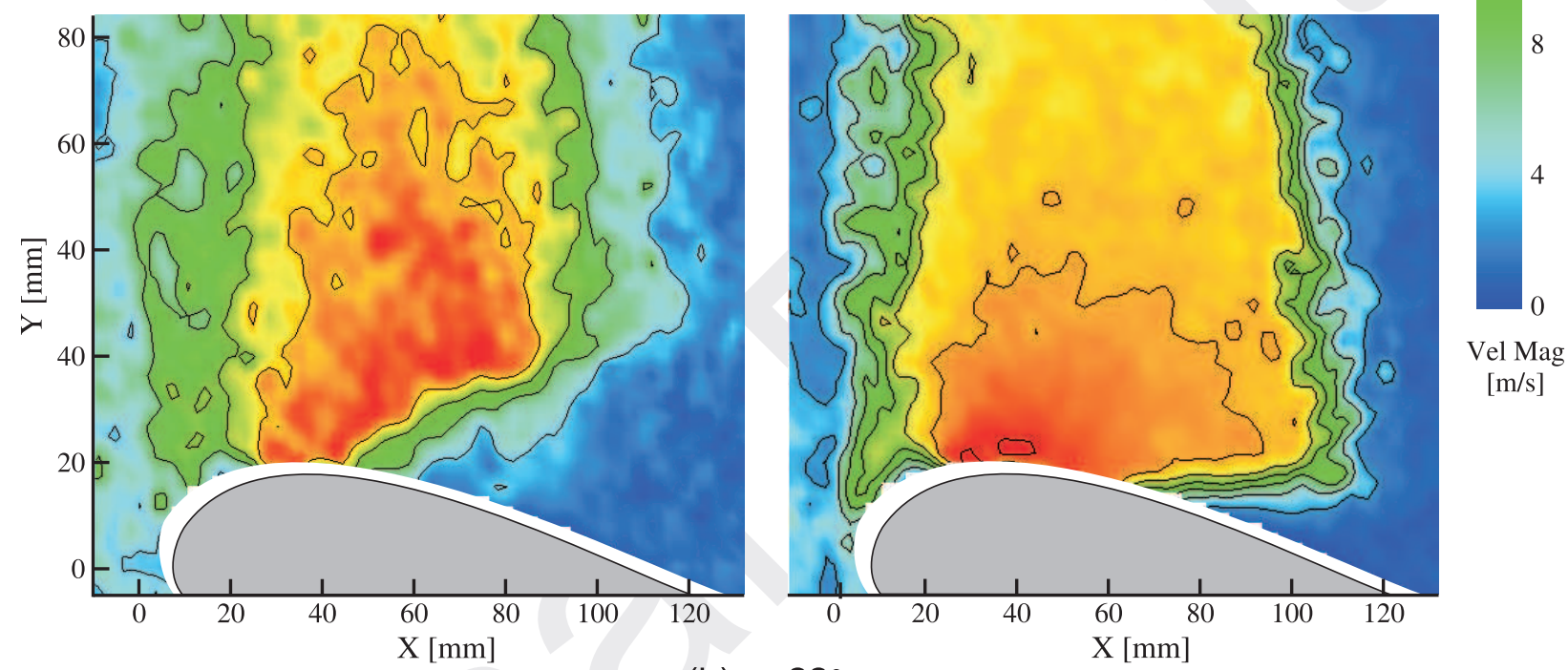

FIG. 8: Velocity contours on the leading edge portion of the upper surface of the aerofoil at $\alpha=$, (a) $17^{\circ}$, (b) $22^{0}$ 

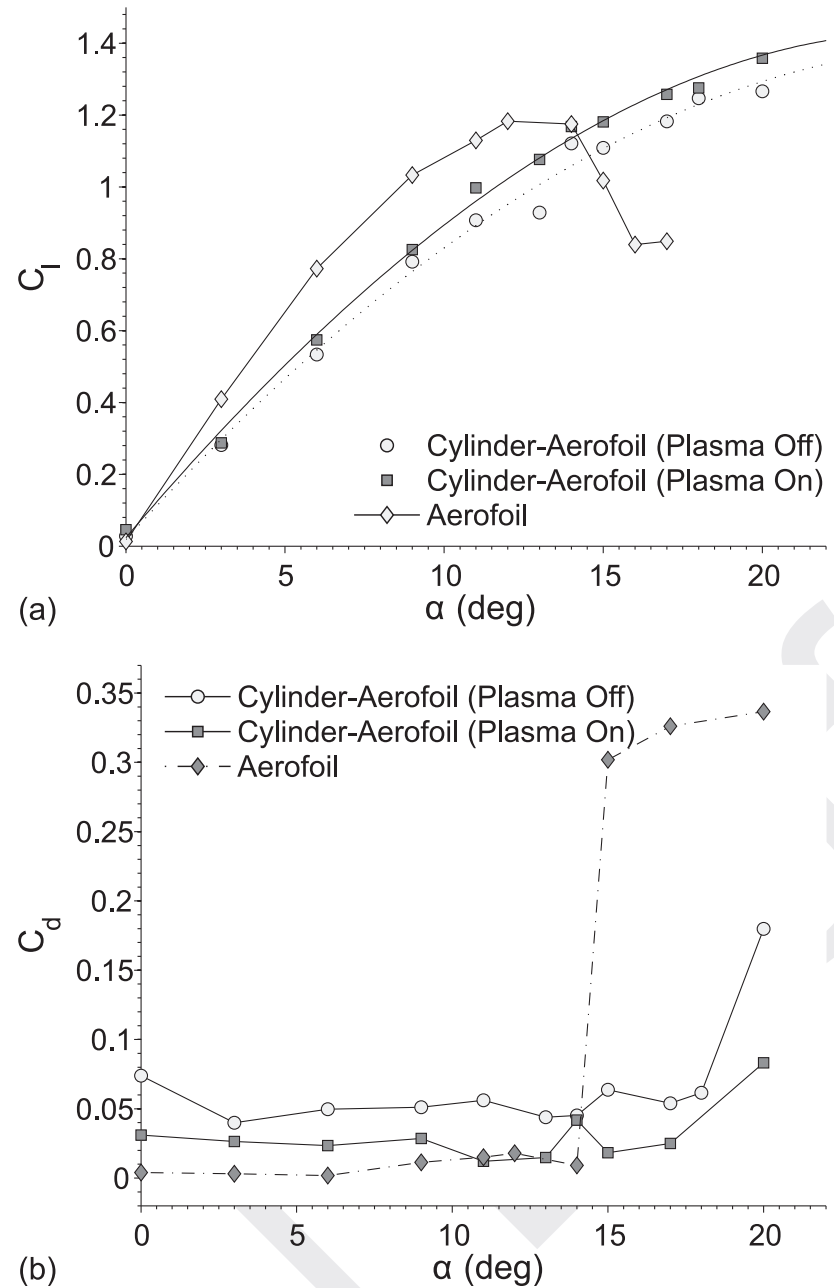

FIG. 9: Lift coefficient (a), and drag coefficient (b) 

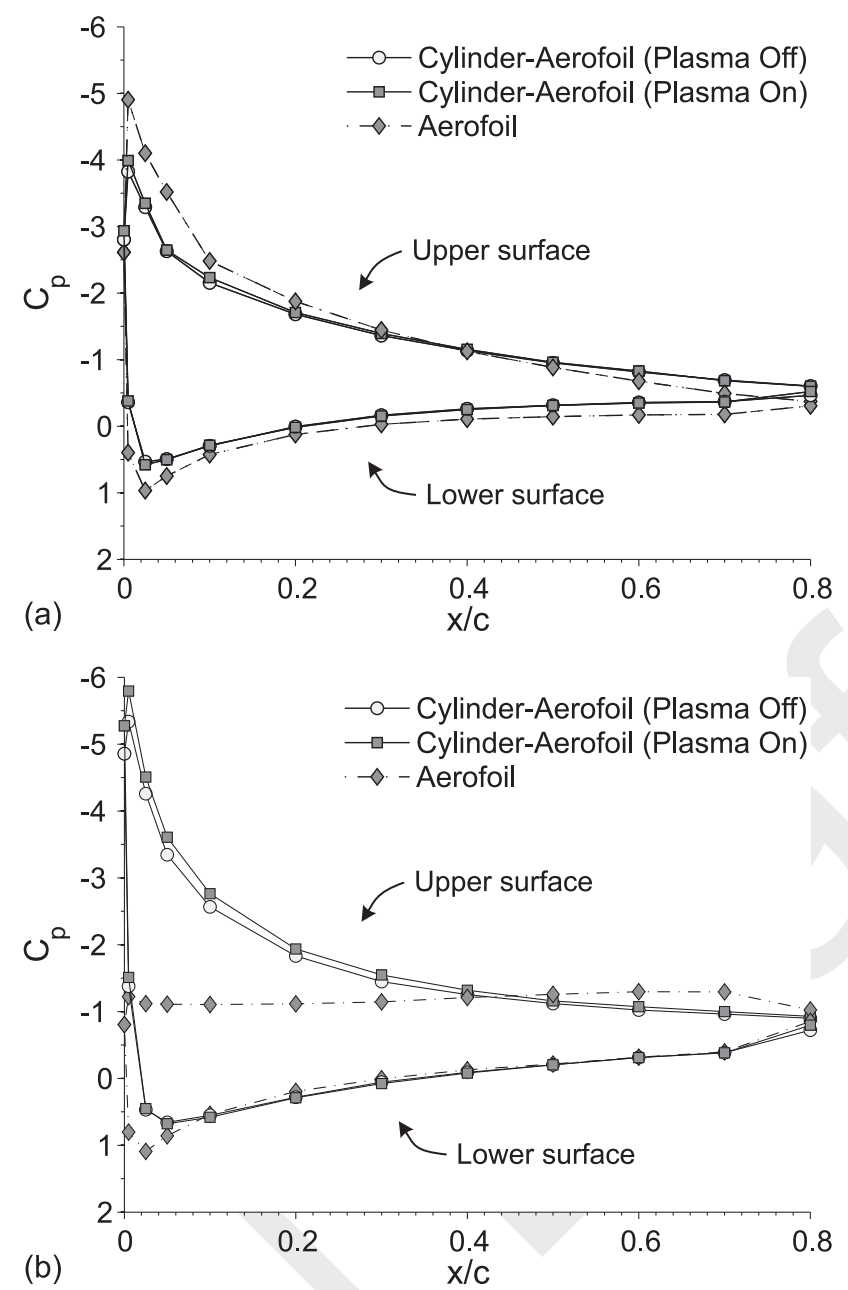

FIG. 10: Pressure distribution on aerofoil surface for $\alpha=$ (a) $11^{\circ}$ (pre-stall), and (b) $17^{\circ}$ (post stall) 